\title{
14. On a Characteristic Property of Completely Normal Spaces
}

\author{
By Takeshi INOKumA \\ Gunma University \\ (Comm. by K. Kunugi, M.J.A., Feb. 18, 1955)
}

Let $H$ be a topological space. We shall consider the following condition:

(V) For any two subsets $X_{1}$ and $X_{2}$ of $H$, there exist two closed sets $H_{1}$ and $H_{2}$ such that

$$
\begin{gathered}
H=H_{1} \smile H_{2} \\
H_{1} \frown H_{2} \frown\left(\bar{X}_{1} \smile \bar{X}_{2}\right)=\bar{X}_{1} \frown \bar{X}_{2} \\
\bar{X}_{i} \subset H_{i}, \quad i=1,2 .
\end{gathered}
$$

Here the bar means the closure operation.

As is well known, the metrizability of $H$ implies (V) and (V) implies the normality of $H$. However it seems that a closer relation between $(\mathrm{V})$ and the separation axioms has not been given in the literature. ${ }^{1)}$

The object of this note is to show that a topological space $H$ is completely normal if and only if it satisfies the condition $(\mathrm{V})$.

1. First we shall prove

Lemma 1. The conditions (1), (2), and (3) imply (1), (3), and (2') below:

and conversely.

$$
H_{i} \frown\left(\bar{X}_{1} \smile \bar{X}_{2}\right) \subset \bar{X}_{i}, \quad i=1,2,
$$

Proof. It is obvious that (1), (2'), and (3) imply (2); we have only to prove that (1), (2), and (3) imply $\left(2^{\prime}\right)$. Since $H_{1} \frown H_{2} \frown$ $\left(\bar{X}_{1} \smile \bar{X}_{2}\right) \frown \bar{X}_{2}=\bar{X}_{1} \frown \bar{X}_{2} \frown \bar{X}_{2}$ by (2), we have $H_{1} \frown \bar{X}_{2}=\bar{X}_{1} \frown \bar{X}_{2}$ by (3). Therefore $\left(H_{1} \frown \bar{X}_{2}\right) \smile \bar{X}_{1}=\left(\bar{X}_{1} \frown \bar{X}_{2}\right) \smile \bar{X}_{1}$, and hence $H_{1} \frown\left(\bar{X}_{1} \smile \bar{X}_{2}\right) \subset \bar{X}_{1}$. Similarly we have $H_{2} \frown\left(\bar{X}_{1} \smile \bar{X}_{2}\right) \subset \bar{X}_{2}$.

Next we shall recall the definition of completely normal spaces; a topological space $H$ is said to be completely normal if the following condition is satisfied:

For any two subsets $Y_{1}$ and $Y_{2}$ of $H$ such that

$$
\bar{Y}_{1} \frown Y_{2}=Y_{1} \frown \bar{Y}_{2}=0,
$$

1) Cf. A. D. Wallace: Dimensional types, Bull. Amer. Math. Soc., 51, 679-681 (1945). Indeed, he said in his paper "the validity of $(\mathrm{V})$ is a well-known property of metric spaces but we have no reference to its formulation in the literature as an axiom". 
there exist two open sets $K_{1}$ and $K_{2}$ of $H$ with the following properties:

$$
\begin{gathered}
K_{1} \frown K_{2}=0 \\
Y_{i} \subset K_{i}, \quad i=1,2 .
\end{gathered}
$$

Now we shall prove

Theorem 1. A necessary and sufficient condition that a topological space $H$ be completely normal is that $H$ satisfy the condition ( $V)$.

Proof. Sufficiency. Assume that (V) is satisfied. Let $Y_{1}$ and $Y_{2}$ be any two subsets of $H$ satisfying (4). Then we can find two closed sets $H_{1}$ and $H_{2}$ which satisfy (1), (2), and (3) with $X_{1}$ and $X_{2}$ replaced by $Y_{1}$ and $Y_{2}$. Let us set $K_{1}=H-H_{2}$ and $K_{2}=H-H_{1}$. Then $K_{i}$ are open and the validity of (5) is obvious from (1). Next we shall show that (6) holds. Suppose that there exists a point $x$ which is contained in $Y_{1}$ but not in $K_{1}$. Then $x$ is contained in $H_{2}$. Since $x \in Y_{1} \subset H_{1}$ by (3), we have $x \in H_{1} \frown H_{2} \frown\left(\bar{Y}_{1} \smile \bar{Y}_{2}\right)=\bar{Y}_{1} \frown \bar{Y}_{2}$. Thus $x \in Y_{1} \frown \bar{Y}_{2}$, this contradicts to (4). Hence we have $Y_{i} \subset K_{i}$, $i=1,2$. Therefore $H$ is completely normal.

Necessity. Let $X_{1}$ and $X_{2}$ be any two subsets of $H$. We put $Y_{1}=\bar{X}_{2}-\bar{X}_{1}$ and $Y_{2}=\bar{X}_{1}-\bar{X}_{2}$. Then $\bar{Y}_{1} \subset \bar{X}_{2}$ and $\bar{Y}_{2} \subset \bar{X}_{1}$, and hence $\bar{Y}_{i} \frown Y_{j} \subset \bar{X}_{j} \frown\left(\bar{X}_{i}-\bar{X}_{j}\right)=0, i, j=1,2$. Thus (4) is fulfilled. Assume that $H$ is completely normal. Then we can find two open sets $K_{1}$ and $K_{2}$ satisfying (5) and (6). Let us put

$$
K_{i}^{\prime}=K_{i \frown}\left(H-\bar{X}_{i}\right), \quad H_{i}=H-K_{i}^{\prime}, \quad i=1,2 .
$$

It is obvious that $H_{1}$ and $H_{2}$ are closed and satisfy (1) and (3). We shall show that $\left(2^{\prime}\right)$ holds also. Since $Y_{1}=\bar{X}_{2}-\bar{X}_{1} \subset K_{1}$, we have $H-K_{1} \subset\left(H-\bar{X}_{2}\right) \smile \bar{X}_{1}$, and hence $H_{1}=H-K_{1}^{\prime}=\left(H-K_{1}\right) \smile \bar{X}_{1} \subset\left(H-\bar{X}_{2}\right) \smile \bar{X}_{1}$ by (7). Therefore $H_{1} \frown\left(\bar{X}_{1} \smile \bar{X}_{2}\right) \subset\left(\left(H-\bar{X}_{2}\right) \smile \bar{X}_{1}\right) \frown\left(\bar{X}_{1} \smile \bar{X}_{2}\right)=\bar{X}_{1}$. Similarly we obtain $H_{2} \frown\left(\bar{X}_{1} \smile \bar{X}_{2}\right) \subset \bar{X}_{2}$. This shows that if $H$ is completely normal, then $H$ satisfies the condition $(V)$.

Thus Theorem 1 is completely proved.

2. We can extend Theorem 1 as follows:

Theorem 2. If a topological space $H$ is completely normal, then for any $n$ subsets $X_{1}, X_{2}, \ldots, X_{n}$ of $H$, there exist $n$ closed sets $H_{1}$, $H_{2}, \ldots, H_{n}$ such that

$$
\begin{gathered}
H=H_{1} \smile H_{2} \smile \ldots \smile H_{n} \\
H_{i} \frown H_{j} \frown\left(\bar{X}_{i} \smile \bar{X}_{j}\right)=\bar{X}_{i} \frown \bar{X}_{j} \\
\bar{X}_{i} \subset H_{i}, \quad i=1,2, \ldots, n .
\end{gathered}
$$

Proof. We shall prove our theorem by induction on $n$. In case $n=2$, Theorem 2 is true by Theorem 1. Assume that Theorem 2 holds for $n$. Let $X_{1}, X_{2}, \ldots, X_{n+1}$ be $n+1$ subsets of $H$. Then we 
can find $n$ closed sets $H_{1}, H_{2}, \ldots, H_{n-1}$, and $H^{\prime}$ satisfying the conditions of Theorem 2 for $n$ given sets $X_{1}, X_{2}, \ldots, X_{n-1}$ and $X^{\prime}=X_{n} \smile_{n+1}$. Since $H^{\prime}$ is completely normal, by Theorem 1 we can select two closed subsets $H_{n}$ and $H_{n+1}$ of $H^{\prime}$ which satisfy (V) for the two given subsets $X_{n}$ and $X_{n+1}$ of $H^{\prime}$. We shall show that these $H_{1}, H_{2}, \ldots$, $H_{n+1}$ fulfill the conditions of Theorem 2 for $X_{1}, X_{2}, \ldots, X_{n+1}$. The conditions $\left(1_{n}\right)$ and $\left(3_{n}\right)$ hold trivially. To prove the equality $H_{i} \frown H_{n} \frown\left(\bar{X}_{i} \smile \bar{X}_{n}\right)=\bar{X}_{i} \frown \bar{X}_{n}$ for $i<n$, let $x \in H_{i} \frown H_{n} \frown\left(\bar{X}_{i} \smile \bar{X}_{n}\right)$. Then we have $x \in H_{i} \frown H^{\prime} \frown\left(\bar{X}_{i} \smile \overline{X_{n} \smile X_{n+1}}\right)=\overline{X_{i} \frown \bar{X}_{n} \smile \bar{X}_{n+1}}$. By Lemma 1 we obtain $x \in H_{n} \frown\left(\overline{X_{n} \smile X_{n+1}}\right) \subset \bar{X}_{n}$. Therefore $x \in \bar{X}_{i} \frown \bar{X}_{n}$ and hence $H_{i} \frown H_{n} \frown\left(\bar{X}_{i} \smile \bar{X}_{n}\right) \subset \bar{X}_{i} \frown \bar{X}_{n}$. Since it is obvious that $H_{i \frown H_{n}}\left(\bar{X}_{i} \smile \bar{X}_{n}\right)$ $\supset \bar{X}_{i} \frown \bar{X}_{n}$, we have $H_{i} \frown H_{n} \frown\left(\bar{X}_{i} \smile \bar{X}_{n}\right)=\bar{X}_{i} \frown \bar{X}_{n}$. Since the above argument holds equally for $H_{n+1}$, the proof is completed.

3. In Theorem 2 the number $n$ is, of course, assumed to be finite. For an infinite number of sets $X_{1}, X_{2}, \ldots$, a similar proposition does not hold in general, as is shown by the following example.

Let $H$ be Euclidian space of dimension 1 , and $X_{1}, X_{2}, \ldots$ be an infinite family of all the subsets $X_{i}$ of $H$ such that $X_{i}$ consists of only one rational point. Then there is no family of closed sets $H_{1}, H_{2}, \ldots$ of $H$ with the following property:

$$
\left\{\begin{array}{l}
H=H_{1} \smile H_{2} \smile \ldots \\
H_{i} \frown H_{j} \frown\left(\bar{X}_{i} \smile \bar{X}_{j}\right)=\bar{X}_{i} \frown \bar{X}_{j} \\
\bar{X}_{i} \subset H_{i}, \quad i=1,2, \ldots .
\end{array}\right.
$$

In this case $H$ is, of course, a completely normal topological space.

Proof. Suppose that there is a desired family $\left\{H_{1}, H_{2}, \ldots\right\}$. Since $H_{i} \frown\left(\bar{X}_{i} \smile \bar{X}_{j}\right) \subset \bar{X}_{i}$, the number of rational points contained in $H_{i}$ is one. The set $R_{0}$ of all irrational points is equal to $\smile\left\{H_{i}-X_{i}\right\}=$ $\smile\left\{H_{i} \frown\left(H-X_{i}\right)\right\}$ and hence $R_{0}$ is an $F_{\sigma}$-set, for $H$ is closed and $H-X_{i}$ is open. Since $R_{0}$ is a $G_{\delta}$-set, it is $F_{\sigma}$ and $G_{\delta}$ at the same time, that is, $R_{0}$ is a developable set in the sense of C. Kuratowski. On the other hand $R_{0}$ is a frontier set (ensemble frontier, i.e. $\overline{H-R_{0}}=H$ ) and hence $R_{0}$ is non dense (i.e. $\overline{H-\overline{R_{0}}}=H$ ). This is a contradiction.

4. We have just obtained the consequence that even in Euclidian space of dimension 1 , the property $\left(\mathrm{V}^{*}\right)$ does not hold in general. So we are led to the consideration of the case where $\left\{X_{1}, X_{2}, \ldots\right\}$ is locally finite.

Theorem 3. If a topological space $H$ is completely normal and fully normal, then for any locally finite family of subsets $X_{1}, X_{2}, \ldots$ of $H$, there exists a family of closed sets $H_{1}, H_{2}, \ldots$ with the property $\left(V^{*}\right)$. 
Proof. Since the family $\left\{X_{\alpha}\right\}$ is locally finite, the family $\left\{\bar{X}_{\alpha}\right\}$ is also locally finite. So we assume $X_{\alpha}$ to be closed and shall prove this theorem for the family of closed sets $\left\{X_{\alpha}\right\}$. For any point $x$ of $H$, there exists a neighbourhood $U$ of $x$ which intersects only a finite number of the sets of $\left\{X_{\alpha}\right\}$. There corresponds a neighbourhood $V$ of $x$ such that $U \supset \bar{V} \supset V$, for $H$ is regular. Thus we obtain an open covering $\{V\}$ such that the closure of any open set of $\{V\}$ intersects only a finite number of sets of $\left\{X_{u}\right\}$. Since $H$ is fully normal, we can find a locally finite open covering $\left\{W_{\beta}\right\}$ which is a refinement of $\{V\}$. Then $\left\{\bar{W}_{\beta}\right\}$ is a locally finite closed covering which is a refinement of $\{\bar{V}\}$. For each $\beta$, let $\left\{X_{i} ; i \in \Gamma_{(\beta)}\right\}$ be the family of the sets $X_{i}$ intersecting $\bar{W}_{\beta}$ (here $\Gamma_{(\beta)}$ is finite). For $\left\{X_{i} \cap \bar{W}_{\beta} ; i \in \Gamma_{(\beta)}\right\}$ we can obtain $\left\{H_{\imath \beta} ; i \in \Gamma_{(\beta)}\right\}$ such that

$$
\begin{gathered}
\bar{W}_{\beta}=\smile\left\{H_{i \beta} ; i \in \Gamma_{(\beta)}\right\}, \\
H_{i \beta} \frown H_{j \beta} \frown\left(\left(X_{i} \frown \bar{W}_{\beta}\right) \smile\left(X_{j} \frown \bar{W}_{\beta}\right)\right)=\left(X_{i} \frown \bar{W}_{\beta}\right) \frown\left(X_{j} \frown \bar{W}_{\beta}\right), \\
X_{i \frown \bar{W}_{\beta} \subset H_{i \beta},}
\end{gathered}
$$

by Theorem 2. Let us set $H_{i}={ }_{\beta}\left\{H_{i \beta}\right\}$ where the sum extends over $\beta$ such that $i \in \Gamma_{(\beta)}$. Then $H_{i}$ is clearly closed. It is obvious that $X_{i} \subset H_{i}$ and $H=\smile\left\{H_{\alpha}\right\}$. We shall show $H_{i} \frown\left(X_{i} \smile X_{j}\right) \subset X_{i}$. Let $x$ be any point of $H_{i} \frown\left(X_{i} \smile X_{j}\right)$. Then for some $\beta, x \in H_{\imath \beta}$. Since $H_{i \beta} \subset \bar{W}_{\beta}, \quad x \in H_{i \beta} \frown\left(\left(X_{i} \frown \bar{W}_{\beta}\right) \smile\left(X_{j} \frown \bar{W}_{\beta}\right)\right)$ and hence by Lemma 1 $x \in\left(X_{i} \frown \bar{W}_{\beta}\right)$ and consequently $x \in X_{i}$. Therefore $H_{i} \frown\left(X_{i} \smile X_{j}\right) \subset X_{i}$. The proof is completed. 